\section{Prevalência de adultos e idosos insuficientemente ativos moradores em áreas de unidades básicas de saúde com e sem Programa Saúde da Família em Pernambuco, Brasil}

\author{
Physical inactivity among adults and elderly living in \\ areas covered by primary healthcare units with \\ and without the Family Health Program \\ in Pernambuco State, Brazil
}

João Guilherme Bezerra Alves 1 Fernando Vinholes Siqueira 2 José Natal Figueiroa 1 Luiz Augusto Facchini 3 Denise Silva da Silveira ${ }^{3}$ Roberto Xavier Piccini 3 Elaine Tomasi 4 Elaine Thumé 5 Pedro C. Hallal 6,7

\footnotetext{
${ }^{1}$ Instituto de Medicina Integral Prof. Fernando Figueira, Recife, Brasil. 2 Centro de Ciências da Vida e da Saúde, Universidade Católica de Pelotas, Pelotas, Brasil.

3 Departamento de Medicina Social, Universidade Federal de Pelotas, Pelotas, Brasil. 4 Programa de Pós-graduação em Saúde e Comportamento, Universidade Católica de Pelotas, Pelotas, Brasil. 5 Faculdade de Enfermagem e Obstetrícia, Universidade Federal de Pelotas, Pelotas, Brasil.

6 Programa de Pós-graduação em Epidemiologia, Universidade Federal de Pelotas, Pelotas, Brasil. 7 Programa de Pós-graduação em Educação Física, Universidade Federal de Pelotas, Pelotas, Brasil.

Correspondência J. G. B. Alves Instituto de Medicina Integral Prof. Fernando Figueira. Rua dos Coelhos 300, Recife, PE 50070-550, Brasil. joaoguilhermealves@ hotmail.com
}

\begin{abstract}
The epidemiological transition in Brazil has been explained partially by the low levels of physical activity. However, few studies have explored physical inactivity in low-income population groups Within this context, primary healthcare units gain strategic importance. This article describes the prevalence of sedentary lifestyle and associated factors in a cross-sectional study, including 1,018 adults and 1,010 elderly adults in 10 cities in Pernambuco State. Prevalence of sedentary lifestyle was 37.8\% (95\%CI: 34.0-40.2) in adults and 68.3\% (95\%CI: 65.3-71.3) in the elderly. Identification of physical activity as the most important factor for good health was associated with sedentary lifestyle in elderly adults. A lower proportion of sedentary lifestyle among non-elderly adults was associated with good self-reported health status. We concluded that sedentary lifestyle prevalence is high in elderly and non-elderly people covered by primary healthcare units in Pernambuco State as compared to the South of Brazil.
\end{abstract}

Motor Activity; Health Centers; Adult; Aged

\section{Introdução}

O sedentarismo eleva o risco de doenças crônicas, especialmente as afecções cardiovasculares, a diabetes tipo 2, o câncer de mama e o de cólon 1,2. A Organização Mundial da Saúde (OMS) 3 estima que o sedentarismo seja responsável, anualmente no mundo, por 1,9 milhões de mortes. Assim, o aumento da atividade física, particularmente entre os segmentos populacionais com maiores prevalências de sedentarismo, representa hoje uma das principais políticas de saúde pública 4. A ratificação da Estratégia Global em Alimentação, Atividade Física e Saúde da OMS, pela Assembléia de Saúde Mundial em maio de 2004, enfatiza o fato de que o sedentarismo é um grande problema de saúde pública, tanto nos países desenvolvidos, quanto nos países em desenvolvimento 5 .

Os países pobres e em desenvolvimento, como o Brasil, vêm apresentando recentemente, importante aumento na incidência de doenças crônicas não transmissíveis 6,7,8. A transição epidemiológica em nosso país tem sido, em parte, explicada pelos baixos níveis de atividade física da população e o consumo de dietas hipercalóricas. Entretanto, poucos estudos têm explorado o nível de atividade física em populações de baixa renda, sendo que uma recente revisão sistemática mostrou haver uma grande disparidade regional nas pesquisas em epidemiologia da atividade física, com clara predominância de estudos nas 
regiões Sul e Sudeste 9 . No Brasil, o sedentarismo apresenta elevada prevalência, especialmente nas regiões menos desenvolvidas como o Nordeste 10. Diminuir o sedentarismo e promover estilos de vida saudáveis com a participação da atenção básica à saúde e seus profissionais pode representar um grande impacto na melhoria dos índices de saúde populacional e nos custos relacionados à gestão dos serviços 11 .

Com a meta de reorganizar a atenção básica à saúde fortalecendo o enfoque preventivo, o Ministério da Saúde implantou em 1994 o Programa Saúde da Família (PSF), seguindo os princípios de universalidade, equidade e integralidade do Sistema Único de Saúde (SUS). Atualmente, o PSF está implantado em várias unidades básicas de saúde (UBS) no Estado de Pernambuco, enquanto outras ainda permanecem com o modelo tradicional, o que propicia um cenário favorável para a avaliação da Estratégia Saúde da Família (ESF) na promoção da saúde e prevenção de doenças crônicas não transmissíveis como a prática de atividade física, especialmente para a população de idosos, segmento populacional que mais vem crescendo no Brasil nas últimas décadas. Ressalte-se ainda a escassez de informações sobre o nível de atividade física no Estado de Pernambuco, com exceção da cidade do Recife, especialmente em populações de baixa renda e adstritas às UBS, conforme pesquisa nos bancos de dados da SciELO e MEDLINE. Este estudo tem o objetivo de estimar e identificar, respectivamente, a prevalência e fatores associados com o sedentarismo em populações de adultos e idosos residentes em áreas de abrangência de UBS de municípios do Estado de Pernambuco.

\section{Métodos}

Foi realizado um estudo de delineamento transversal com uma amostra de indivíduos adultos (30 a 64 anos) e idosos (65 ou mais), residentes nas áreas de abrangência das UBS, dos dez municípios com mais de 100 mil habitantes do Estado de Pernambuco. Os municípios incluídos no estudo fazem parte do Lote Nordeste 2 do Estudo de Linha de Base do Projeto de Expansão e Consolidação da Saúde da Família (PROESF).

Uma amostra aleatória de UBS foi sorteada em cada um dos municípios do Estado de Pernambuco, com diferentes modalidades de atenção básica - PSF e tradicional. O sorteio das UBS foi proporcional ao tamanho da rede básica de cada município. A partir de listas produzidas pelos municípios, as UBS foram selecionadas na razão de duas UBS com PSF para uma UBS tradicional. A seleção da amostra nos municípios foi proporcional à capacidade instalada de sua rede básica. No Nordeste, a amostra foi constituída de 79 UBS de PSF e 41 tradicionais. No Estado de Pernambuco a amostra ficou constituída por 39 UBS do tipo PSF e 18 do modelo tradicional. As UBS sorteadas orientaram a seleção da amostra de adultos e idosos da área de abrangência dos serviços de forma sistemática e independente. A estratégia para delimitação da área de abrangência incluiu a obtenção prévia de seu mapa, e uma estimativa populacional a partir das áreas censitárias do Instituto Brasileiro de Geografia e Estatística (IBGE). A localização dos indivíduos na área delimitada ocorreu através de amostragem sistemática e incluiu apenas um indivíduo por domicílio. Nos domicílios onde mais de um indivíduo era elegível, aquele de mais idade era o selecionado. Para a aplicação do questionário, foram capacitados 15 supervisores de campo que realizaram a coleta de dados após período de treinamento.

Com a amostra obtida de 1.018 adultos e de 1.010 idosos, a margem de erro para a estimativa de prevalência de sedentarismo foi de 1,5 pontos percentuais, utilizando-se uma prevalência de inatividade física de $40 \%$. Para avaliação de associações, o estudo teve poder de $80 \%$ para detectar como significativas razões de prevalência (RP) de 1,2 ou maiores, para exposições que afetam de $10 \%$ a $90 \%$ da população, com nível de $95 \%$ de confiança.

Unidades básicas tradicionais foram consideradas aquelas que se caracterizavam pela presença na equipe de médicos especialistas (clínico, pediatra e gineco-obstetra), enfermeiro, auxiliar de enfermagem e pessoal administrativo para apoio técnico. Muitas destas unidades contam com o apoio esporádico de outras especialidades profissionais (dentista, nutricionista, fisioterapeuta) e mesmo médicas. A população atendida caracteriza-se por ser espontânea ou devido a encaminhamento de outros serviços. As unidades de PSF, ao contrário, são formadas por equipes de saúde da família, que incluem médico generalista, enfermeiro, auxiliares de enfermagem e agentes comunitários, responsáveis pelo cadastramento e acompanhamento da população residente na área de abrangência do serviço 12 .

O nível de atividade física dos adultos e idosos foi avaliado utilizando-se a versão curta do Questionário Internacional de Atividade Física (IPAQ) ${ }^{13}$. Este instrumento avalia atividades físicas realizadas no tempo de lazer, deslocamento, serviços domésticos e atividades ocupacionais. Mede a freqüência e duração das atividades físicas moderadas, vigorosas e caminhadas realizadas na última semana por pelo menos dez minutos contínuos incluindo exercício, esportes 
e atividades físicas ocupacionais e recreacionais realizadas em casa, no tempo livre, como meio de transporte e no lazer. Um escore de atividade física em minutos por semana foi construído, somando-se os minutos despendidos em caminhada e atividades de intensidade moderada com os minutos despendidos em atividades de intensidade vigorosa multiplicados por dois. Esta estratégia visa considerar as diferentes intensidades de cada atividade, e está de acordo com as recomendações atuais quanto à prática de atividade física 14,15. Um escore abaixo de 150 minutos por semana foi o ponto de corte para classificar os indivíduos como sedentários 16. O questionário utilizado no estudo foi padronizado e pré-testado.

As variáveis independentes incluídas nesta análise foram: (a) sexo; (b) idade; (c) cor da pele (através da percepção do entrevistador); (d) escolaridade (anos de estudo com aprovação); (e) tabagismo (nunca fumou, ex-fumante, fumante atual); (f) nível econômico (segundo classificação da Associação Brasileira de Empresas de Pesquisa (ABEP) que leva em consideração a posse de bens de consumo, grau de instrução do chefe de família e presença de empregada mensalista; http://www.abep.org/codigosguias/abep.cceb. pdf, acessado em 12/Jun/2007); (g) situação conjugal; (h) renda familiar per capita (em salários mínimos); (i) trabalho remunerado no último mês (não/licença ou nunca trabalhou, e sim); (j) autopercepção de saúde (excelente, muito boa, boa, regular, ruim); (k) identificação da atividade física, todo movimento corporal voluntário humano e que resulta num gasto energético acima dos níveis de repouso, como um dos três fatores mais importantes para a manutenção da saúde. Os fatores considerados foram: manter uma alimentação saudável, fazer exercício físico regularmente, não tomar bebidas alcoólicas em excesso, realizar consultas médicas regularmente, não fumar, manter o peso ideal e controlar ou evitar o estresse. A partir disso foi possível verificar se a pessoa identificou ou não a atividade física como fator importante para a manutenção da saúde; (l) prescrição de atividade física em consulta realizada no último ano (não/sim).

Seguiu-se um modelo hierárquico de análise dividido em quatro níveis: no primeiro nível foram incluídas as variáveis independentes (a, b, c, d, e f, g, h); no segundo nível ficou apenas a variável independente (i); no terceiro nível foram incluídas as variáveis independentes (j) e (k); no quarto nível, a variável independente (l). Tal modelo de análise baseou-se em modelo teórico elaborado previamente à coleta de dados. As variáveis foram incluídas no modelo nível por nível, sendo retiradas aquelas com valor de p superior a
0,20 . As demais foram mantidas no modelo para controle de confundimento. Ajustou-se o efeito de cada preditor sobre o desfecho apenas para as exposições que se encontravam no mesmo nível ou em níveis superiores no modelo de análise. $\mathrm{O}$ nível de significância usado foi de $5 \%$.

As análises descritivas incluíram cálculos de proporções e respectivos intervalos de 95\% de confiança (IC95\%). Na análise bruta, a prevalência de sedentarismo foi calculada para cada grupo das variáveis independentes, e o nível de significância foi testado usando o teste de Wald para heterogeneidade ou tendência linear. A análise ajustada foi realizada por regressão de Poisson 17 com cálculo robusto de razões de prevalência, IC95\%, e valores de significância usando os mesmos testes descritos acima. Todas as análises levaram em consideração o desenho amostral, e foi utilizado um modelo hierárquico de determinação do desfecho ${ }^{18}$. O modelo incluiu as variáveis independentes (a, c, d, e, f, g, h) no primeiro nível. A variável (i) foi incluída no segundo nível, enquanto o terceiro nível incluiu as variáveis $(\mathrm{j}, \mathrm{k})$ e o quarto incluiu a variável independente (l).

O Comitê de Ética da Faculdade de Medicina da Universidade Federal de Pelotas aprovou o protocolo do estudo, e obteve-se consentimento informado de todos os participantes. Mais detalhes sobre a metodologia do estudo pode ser lida em outras publicações 19,20.

\section{Resultados}

No Estado de Pernambuco, a prevalência de sedentarismo entre os adultos foi $37,1 \%$ (IC95\%: 34,0-40,2) e entre os idosos, 68,3\% (IC95\%: 65,3$71,3 \%)$. A Tabela 1 descreve a amostra de adultos e idosos conforme as variáveis biológicas e sócio-demográficas incluídas na investigação. A proporção de mulheres foi maior em ambos os grupos etários e a média de idade nos adultos foi de 45,2 anos (desvio-padrão - DP = 9,6 anos) enquanto nos idosos foi de 74,1 anos (DP = 7,3 anos). Tanto na amostra de adultos quanto na amostra de idosos, a categoria de renda familiar per capita de até um salário mínimo foi a de maior prevalência. Um pouco mais da metade dos adultos consideraram a saúde pessoal como regular ou ruim, enquanto entre os idosos, esse percentual chegou a $70 \%$. O percentual de indivíduos que classificou a atividade física como um dos três comportamentos mais importantes para a manutenção da saúde foi igual a $61,2 \%$ entre os adultos e $46,7 \%$ entre os idosos. No primeiro grupo, 25,7\% receberam prescrição de atividade física na UBS no último ano, enquanto no segundo grupo esse valor foi $30,3 \%$. 
Descrição das variáveis biológicas e sócio-demográficas em adultos e idosos na população de abrangência das unidades básicas de saúde (UBS) de municípios do Estado de Pernambuco, Brasil, 2005.

\begin{tabular}{|c|c|c|c|c|}
\hline \multirow[t]{2}{*}{ Variáveis } & \multicolumn{2}{|c|}{ Adultos } & \multicolumn{2}{|c|}{ Idosos } \\
\hline & $\%$ & $\mathbf{n}$ & $\%$ & $\mathbf{n}$ \\
\hline Sexo & & 1.018 & & 1.010 \\
\hline Masculino & 46,6 & & 37,9 & \\
\hline Feminino & 53,4 & & 62,1 & \\
\hline Idade (anos) & & 1.017 & & 1.010 \\
\hline $30-40$ & 36,5 & & - & \\
\hline $41-50$ & 32,7 & & - & \\
\hline $51-64$ & 30,8 & & - & \\
\hline $65-70$ & - & & 37,9 & \\
\hline $71-75$ & - & & 25,1 & \\
\hline $76-79$ & - & & 14,3 & \\
\hline 80 ou + & - & & 22,7 & \\
\hline Cor da pele & & 1.013 & & 1.006 \\
\hline Branca & 52,5 & & 55,4 & \\
\hline Não branca & 47,5 & & 44,6 & \\
\hline Escolaridade & & 1.014 & & 985 \\
\hline Até primeiro grau incompleto & - & & 36,8 & \\
\hline Primeiro grau completo ou mais & - & & 63,2 & \\
\hline Não estudou & 19,6 & & - & \\
\hline Fundamental incompleto & 43,0 & & - & \\
\hline Fundamental completo & 9,4 & & - & \\
\hline Médio incompleto & 5,3 & & - & \\
\hline Médio completo & 18,6 & & - & \\
\hline Superior & 4,1 & & - & \\
\hline Tabagismo & & 1.018 & & 1.008 \\
\hline Não, nunca fumou & 52,7 & & 44,4 & \\
\hline Já fumou & 26,2 & & 42,4 & \\
\hline Fumante atual & 21,1 & & 13,2 & \\
\hline Padrão de consumo sócio-econômico * & & 974 & & 910 \\
\hline$A, B$ ou $C$ & 29,7 & & 17,2 & \\
\hline $\mathrm{D}$ & 33,9 & & 32,1 & \\
\hline$E$ & 36,4 & & 50,7 & \\
\hline Situação conjugal & & 1.018 & & 1.000 \\
\hline Casado(a)/Com companheiro(a) & 72,1 & & 41,1 & \\
\hline Viúvo(a) & 7,2 & & 44,8 & \\
\hline Separado(a)/Divorciado(a) & 10,4 & & 7,5 & \\
\hline Solteiro(a) & 10,3 & & 6,6 & \\
\hline Renda familiar per capita (salários mínimos) & & 1.017 & & 1.007 \\
\hline Sem renda & 10,4 & & 6,3 & \\
\hline Até 1 & 72,5 & & 81,4 & \\
\hline $1,01-2$ & 12,6 & & 9,1 & \\
\hline $2,01-3$ & 2,5 & & 1,7 & \\
\hline 3 ou + & 2,0 & & 1,5 & \\
\hline Trabalho remunerado no último mês & & 1.017 & & 1.009 \\
\hline Não & 57,1 & & 95,9 & \\
\hline Sim & 42,9 & & 4,1 & \\
\hline
\end{tabular}


Tabela 1 (continuação)

\begin{tabular}{|c|c|c|c|c|}
\hline \multirow[t]{2}{*}{ Variáveis } & \multicolumn{2}{|c|}{ Adultos } & \multicolumn{2}{|c|}{ Idosos } \\
\hline & $\%$ & $\mathrm{n}$ & $\%$ & $\mathrm{n}$ \\
\hline Autopercepção da saúde & & 1.016 & & 995 \\
\hline Excelente & 5,6 & & 2,1 & \\
\hline Muito boa & 4,6 & & 1,8 & \\
\hline Boa & 33,7 & & 26,1 & \\
\hline Regular & 43,9 & & 46,0 & \\
\hline Ruim & 12,2 & & 24,0 & \\
\hline \multicolumn{5}{|c|}{ Identificação da atividade física como } \\
\hline \multicolumn{5}{|c|}{ benefício para a saúde } \\
\hline Não & 38,8 & & 53,3 & \\
\hline Sim & 61,2 & & 46,7 & \\
\hline Prescrição de atividade física no & & 787 & & 884 \\
\hline \multicolumn{5}{|l|}{ último ano } \\
\hline Não & 74,3 & & 69,7 & \\
\hline Sim & 25,7 & & 30,3 & \\
\hline
\end{tabular}

* Segundo classificação da Associação Brasileira de Empresas de Pesquisa (http://www.abep.org/codigosguias/abep.cceb. pdf, acessado em 12/Jun/2007).

As Tabelas 2 e 3 apresentam a associação bruta e ajustada entre sedentarismo e as variáveis biológicas e sócio-demográficas mensuradas em idosos residentes em áreas de abrangência de UBS tanto do tipo tradicional quanto do tipo PSF. Entre os idosos atendidos nas unidades básicas do modelo tradicional, foi encontrada uma prevalência de sedentarismo igual a 68,9\% (IC95\%: 63,5-74,0). Na análise ajustada, o sedentarismo apresentou uma associação significante apenas com as variáveis idade, autopercepção da saúde e identificação da atividade física como benefício para a saúde. Houve maior prevalência de sedentarismo nos idosos com idade a partir de 76 anos (RP = 1,1; IC95\%: 1,0-1,2; p = 0,004), nos que tinham escolaridade a partir do primeiro grau completo (RP = 1,1; IC95\%: 1,0-1,3; p = 0,01) e naqueles com uma autopercepção ruim da saúde (RP = 1,1; IC95\%: 1,0-1,3; p = 0,02). Por outro lado, a prevalência de sedentarismo foi menor nos idosos que identificaram a atividade física como benefício para a saúde $(\mathrm{RP}=0,8$; IC95\%: 0,7-0,9; $\mathrm{p}=0,01)$. Nos idosos atendidos nas unidades básicas tipo PSF, a prevalência de sedentarismo foi 68\% (IC95\%: 64,2-71,7). Na análise ajustada, o sedentarismo apresentou associação significante apenas com as variáveis idade e autopercepção da saúde. O sedentarismo foi mais prevalente entre os idosos com idade a partir de 76 anos (RP = 1,1; IC95\%: 1,1-1,2; p < 0,001), e os que tinham autopercepção regular ou ruim da saúde $(\mathrm{RP}=$ 1,1 ; IC95\%: 1,0-1,1; $\mathrm{p}=0,02$ ).

Também foi analisada a ocorrência de sedentarismo nos adultos, segundo o modelo de aten- ção utilizado (Tabelas 4 e 5). Nos adultos atendidos nas unidades básicas do modelo tradicional, foi encontrada uma prevalência de sedentarismo de 41,7\% (IC95\%: 36,0-47,5). Na análise ajustada, o sedentarismo apresentou associação significante com a cor da pele, escolaridade e padrão de consumo sócio-econômico. O sedentarismo foi menos prevalente entre as mulheres $(\mathrm{RP}=$ 0,7; IC95\%: 0,5-1,0; $p=0,03$ ), entre os de cor nãobranca (RP = 0,7; IC95\%: 0,5-1,0; $p=0,024)$, os de escolaridade em nível de Ensino Médio $(\mathrm{RP}=0,8$; IC95\%: 0,7-1,0; $p=0,005)$, e de padrão de consumo sócio-econômico E (RP = 0,8; IC95\%: 0,7-1,0; $\mathrm{p}=0,02)$. Nos adultos atendidos nas unidades básicas tipo PSF, a prevalência de sedentarismo foi igual a 37,1\% (IC95\%: 33,4-41,0). Na análise ajustada, o sedentarismo apresentou associação significante apenas com o sexo e autopercepção da saúde. O sedentarismo foi menos prevalente entre as mulheres $(\mathrm{RP}=0,6$; IC95\%: 0,5-0,8; $\mathrm{p}<$ $0,001)$ e aqueles que tinham autopercepção ruim da saúde (RP = 1,2; IC95\%: 1,0-1,3; $\mathrm{p}=0,001)$.

\section{Discussão}

A prevalência de sedentarismo observada nos adultos, $37,1 \%$, foi muito similar à observada em outros estudos que também utilizaram a versão curta do IPAQ. O estudo do VIGITEL (Vigilância de Fatores de Risco e Proteção para Doenças Crônicas por Inquérito Telefônico) mostrou uma prevalência na cidade do Recife de $32 \%$ sedentarismo, conceituado como indivíduos que não 
Prevalência de sedentarismo, análise bruta e ajustada em relação ao desfecho sedentarismo em idosos, na população da área de abrangência de unidades básicas de saúde (USB) do Estado de Pernambuco, Brasil. UBS tradicional.

\begin{tabular}{|c|c|c|c|c|c|}
\hline \multirow[t]{2}{*}{ Variáveis } & \multirow[t]{2}{*}{$\%$} & \multicolumn{2}{|c|}{ Análise bruta } & \multicolumn{2}{|c|}{ Análise ajustada } \\
\hline & & $\begin{array}{c}\text { RP } \\
\text { (IC95\%) }\end{array}$ & $\begin{array}{l}\text { Valor } \\
\text { de } p\end{array}$ & $\begin{array}{c}\text { RP } \\
\text { (IC95\%) }\end{array}$ & $\begin{array}{l}\text { Valor } \\
\text { de } p\end{array}$ \\
\hline \multicolumn{6}{|l|}{ Nível 1} \\
\hline Sexo & & & 0,91 & & 0,92 \\
\hline Masculino & 69,3 & 1,0 & & 1,0 & \\
\hline Feminino & 68,7 & $1,0(0,8-1,2)$ & & $1,0(0,8-1,2)$ & \\
\hline Idade (anos) & & & 0,003 * & & 0,004 * \\
\hline $65-70$ & 60,0 & 1,0 & & 1,0 & \\
\hline $71-75$ & 68,2 & $1,1(0,9-1,4)$ & & $1,1(0,9-1,4)$ & \\
\hline $76-79$ & 75,0 & $1,2(1,0-1,6)$ & & $1,3(1,0-1,6)$ & \\
\hline 80 ou + & 79,4 & $1,3(1,1-1,6)$ & & $1,3(1,1-1,6)$ & \\
\hline Cor da pele & & & 0,75 & & 0,43 \\
\hline Branca & 68,0 & 1,0 & & 1,0 & \\
\hline Não branca & 69,6 & $1,0(0,9-1,2)$ & & $1,1(0,9-1,3)$ & \\
\hline Escolaridade & & & 0,07 * & & 0,10 * \\
\hline Até primeiro grau incompleto & 62,3 & 1,0 & & 1,0 & \\
\hline Primeiro grau completo ou mais & 72,3 & $1,2(1,0-1,4)$ & & $1,1(1,0-1,3)$ & \\
\hline Tabagismo & & & 0,35 * & & 0,22 * \\
\hline Não, nunca fumou & 66,9 & 1,0 & & 1,0 & \\
\hline Já fumou & 70,5 & $1,0(0,9-1,2)$ & & $1,0(0,9-1,2)$ & \\
\hline Fumante atual & 73,7 & $1,1(0,9-1,4)$ & & $1,2(0,9-1,4)$ & \\
\hline Padrão de consumo sócio-econômico ** & & & 0,92 * & & 0,41 * \\
\hline$A, B$ ou $C$ & 72,1 & 1,0 & & 1,0 & \\
\hline $\mathrm{D}$ & 66,0 & $0,9(0,7-1,1)$ & & $0,9(0,7-1,1)$ & \\
\hline$E$ & 70,3 & $1,0(0,8-1,2)$ & & $0,9(0,7-1,1)$ & \\
\hline Situação conjugal & & & 0,95 & & 0,87 \\
\hline Casado(a)/Com companheiro(a) & 67,8 & 1,0 & & 1,0 & \\
\hline Viúvo(a) & 70,4 & $1,0(0,9-1,2)$ & & $1,0(0,8-1,2)$ & \\
\hline Separado(a)/Divorciado(a) & 57,1 & $0,8(0,6-1,2)$ & & $0,8(0,5-1,2)$ & \\
\hline Solteiro(a) & 72,7 & $1,1(0,81-1,43)$ & & $1,1(0,8-1,5)$ & \\
\hline Renda familiar per capita (salários mínimos) & & & 0,08 * & & 0,11 * \\
\hline Sem renda & 78,6 & 1,0 & & & \\
\hline Até 1 & 69,9 & $0,9(0,7-1,1)$ & & $0,9(0,7-1,1)$ & \\
\hline $1,01-2$ & 60,0 & $0,8(0,5-1,1)$ & & $0,8(0,5-1,1)$ & \\
\hline $2,01-3$ & 37,5 & $0,5(0,2-1,2)$ & & $0,5(0,2-1,2)$ & \\
\hline 3 ou + & 75,0 & $1,0(0,5-1,7)$ & & $1,0(0,5-1,8)$ & \\
\hline \multicolumn{6}{|l|}{ Nível 2} \\
\hline Trabalho remunerado no último mês & & & 0,29 & & 0,34 \\
\hline Não & 69,6 & 1,0 & & 1,0 & \\
\hline Sim & 55,6 & $0,8(0,5-1,2)$ & & $0,8(0,5-1,3)$ & \\
\hline
\end{tabular}

(continua)

realizam atividade física por pelo menos dez minutos contínuos por semana ${ }^{20}$. Um estudo em Pelotas (Rio Grande do Sul), com o mesmo instrumento aplicado em uma população adulta, observou prevalência de sedentarismo de $41,1 \%$, utilizando o mesmo ponto de corte de $150 \mathrm{mi}$ - nutos por semana 21. Em São Paulo, detectou-se uma freqüência de $46,5 \%$ de sedentarismo nos indivíduos estudados, usando os mesmos critérios do estudo do VIGITEL ${ }^{22}$. Registre-se que os estudos do VIGITEL e o de São Paulo não usaram exatamente o mesmo ponto de corte, o que pode 
Tabela 2 (continuação)

\begin{tabular}{|c|c|c|c|c|c|}
\hline \multirow[t]{2}{*}{ Variáveis } & \multirow[t]{2}{*}{$\%$} & \multicolumn{2}{|c|}{ Análise bruta } & \multicolumn{2}{|c|}{ Análise ajustada } \\
\hline & & $\begin{array}{c}\text { RP } \\
\text { (IC95\%) }\end{array}$ & $\begin{array}{l}\text { Valor } \\
\text { de } p\end{array}$ & $\begin{array}{c}\text { RP } \\
\text { (IC95\%) }\end{array}$ & $\begin{array}{l}\text { Valor } \\
\text { de } p\end{array}$ \\
\hline \multicolumn{6}{|l|}{ Nível 3} \\
\hline Autopercepção da saúde & & & 0,04 * & & 0,02 * \\
\hline Excelente & 71,4 & 1,0 & & 1,0 & \\
\hline Muito boa & 50,0 & $0,9(0,5-1,4)$ & & $0,7(0,4-1,4)$ & \\
\hline Boa & 63,9 & $0,6(0,3-1,4)$ & & $0,6(0,3-1,3)$ & \\
\hline Regular & 64,2 & $0,8(0,6-1,0)$ & & $0,8(0,6-1,0)$ & \\
\hline Ruim & 81,8 & $0,8(0,7-0,9)$ & & $0,8(0,7-1,0)$ & \\
\hline Identificação de atividade física como & & & 0,002 & & 0,01 \\
\hline \multicolumn{6}{|l|}{ benefício para a saúde } \\
\hline Não & 75,8 & 1,0 & & 1,0 & \\
\hline Sim & 57,9 & $0,8(0,6-0,9)$ & & $0,8(0,7-0,9)$ & \\
\hline \multicolumn{6}{|l|}{ Nível 4} \\
\hline Prescrição de atividade física & & & 0,60 & & 0,64 \\
\hline \multicolumn{6}{|l|}{ no último ano } \\
\hline Não & 69,9 & 1,0 & & 1,0 & \\
\hline Sim & 66,0 & $1,0(0,8-1,2)$ & & $0,9(0,8-1,2)$ & \\
\hline
\end{tabular}

RP: razão de prevalência; IC95\%: intervalo de 95\% de confiança.

Nota: níveis correspondentes ao nível da variável no modelo hierárquico. Cada associação foi ajustada para as variáveis de mesmo nível.

* Teste de tendência;

** Segundo classificação da Associação Brasileira de Empresas de Pesquisa (http://www.abep.org/codigosguias/abep.cceb. pdf, acessado em 12/Jun/2007).

justificar a pequena diferença das prevalências de sedentarismo observadas.

Quando comparamos nossos dados com um estudo recente, feito em uma população de favela na cidade do Recife, utilizando o mesmo instrumento, versão curta do IPAQ, e ponto de corte, menos de 150 minutos de atividade física por semana, observamos que nossos índices de sedentarismo foram semelhantes nessa população: $23,4 \%$ e $24,8 \%$, respectivamente 23 . Particularmente em pessoas com elevado nível de pobreza, as atividades ocupacionais e de transporte representam uma proporção substancial das atividades físicas dos indivíduos, o que poderia justificar essa diferença. Já os estudos nos países desenvolvidos têm demonstrado que os níveis de atividade física são inferiores nas populações com níveis sócio-econômicos mais baixos, especialmente a atividade física de lazer 24 .

Ocorre um acentuado declínio do nível de atividade física após os trinta anos de idade, o que tem sido atribuído a adoção de estilo de vida sedentário com o envelhecimento, associado a uma diminuição da aptidão física, que por sua vez é agravada pela inatividade física 25 . Encontramos uma associação positiva da idade com o sedentarismo, a qual é consistente com a literatura 26. A avaliação da atividade física em idosos é complexa e poucos estudos têm sido realizados no Brasil com essa finalidade. $\mathrm{O}$ IPAQ é recomendado para avaliação de atividade física em indivíduos dos 18-64 anos 13. Em um estudo recente realizado em Joaçaba (Santa Catarina, Brasil), que utilizou a versão longa do IPAQ, observou-se um percentual de sedentarismo em idosos (40,7\%) bem inferior ao nosso 27 . Acreditamos que é provável que o maior nível de instrução da população estudada no Estado de Santa Catarina - 80\% sabiam ler e escrever e $11,9 \%$ tinham curso superior - possa justificar essa diferença.

Os resultados dos estudos que relacionam o nível de atividade física total dos indivíduos com o sexo apresentam alguns resultados discordantes. Em nosso, estudo os homens eram mais freqüentemente sedentários. Hallal et al. 21, em Pelotas, não observaram diferenças em relação ao sexo, assim como Baretta et al. 27, em Joaçaba (Santa Catarina). Estudo realizado em favela na cidade do Recife observou um nível de atividade física mais elevado no sexo masculino, habitualmente relacionado ao subemprego ou trabalho "em bico", associado com grande dispêndio energético 23. Matsudo et al. 22, em São Paulo, ob- 
Prevalência de sedentarismo, análise bruta e ajustada em relação ao desfecho sedentarismo em idosos, na população da área de abrangência de unidades básicas de saúde (UBS) do Estado de Pernambuco, Brasil. UBS com Programa Saúde da Família (PSF).

\begin{tabular}{|c|c|c|c|c|c|}
\hline \multirow[t]{2}{*}{ Variáveis } & \multirow[t]{2}{*}{$\%$} & \multicolumn{2}{|c|}{ Análise bruta } & \multicolumn{2}{|c|}{ Análise ajustada } \\
\hline & & $\begin{array}{c}\text { RP } \\
\text { (IC95\%) }\end{array}$ & $\begin{array}{l}\text { Valor } \\
\text { de } p\end{array}$ & $\begin{array}{c}\text { RP } \\
\text { (IC95\%) }\end{array}$ & $\begin{array}{l}\text { Valor } \\
\text { de } p\end{array}$ \\
\hline \multicolumn{6}{|l|}{ Nível 1} \\
\hline Sexo & & & 0,75 & & 0,79 \\
\hline Masculino & 68,7 & 1,0 & & 1,0 & \\
\hline Feminino & 67,5 & $1,0(0,9-1,1)$ & & $1,0(0,9-1,1)$ & \\
\hline Idade (anos) & & & $<0,001$ * & & $<0,001$ * \\
\hline $65-70$ & 56,6 & 1,0 & & 1,0 & \\
\hline $71-75$ & 64,2 & $1,1(1,0-1,3)$ & & $1,1(1,0-1,3)$ & \\
\hline $76-79$ & 79,5 & $1,4(1,2-1,6)$ & & $1,4(1,2-1,6)$ & \\
\hline $80 \mathrm{ou}+$ & 84,5 & $1,5(1,3-1,7)$ & & $1,5(1,3-1,7)$ & \\
\hline Cor da pele & & & 0,72 & & 0,86 \\
\hline Branca & 68,5 & 1,0 & & 1,0 & \\
\hline Não branca & 67,2 & $1,0(0,9-1,1)$ & & $1,0(0,9-1,1)$ & \\
\hline Escolaridade & & & 0,05 & & 0,11 \\
\hline Até primeiro grau incompleto & 62,6 & 1,0 & & 1,0 & \\
\hline Primeiro grau completo ou mais & 70,6 & $1,1(1,0-1,3)$ & & $1,1(1,0-1,2)$ & \\
\hline Tabagismo & & & 0,27 * & & 0,24 * \\
\hline Não, nunca fumou & 64,8 & 1,0 & & 1,0 & \\
\hline Já fumou & 71,1 & $1,1(1,0-1,2)$ & & $1,1(0,9-1,2)$ & \\
\hline Fumante atual & 68,5 & $1,1(0,9-1,2)$ & & $1,1(0,9-1,3)$ & \\
\hline Padrão de consumo sócio-econômico ** & & & 0,61 * & & 0,85 * \\
\hline$A, B$ ou $C$ & 70,5 & 1,0 & & 1,0 & \\
\hline $\mathrm{D}$ & 68,4 & $1,0(0,8-1,1)$ & & $1,0(0,8-1,2)$ & \\
\hline$E$ & 67,5 & $1,0(0,8-1,1)$ & & $1,0(0,8-1,2)$ & \\
\hline Situação conjugal & & & 0,32 & & 0,47 \\
\hline Casado(a)/Com companheiro(a) & 63,4 & 1,0 & & 1,0 & \\
\hline Viúvo(a) & 74,2 & $1,2(1,0-1,3)$ & & $1,1(1,0-1,2)$ & \\
\hline Separado(a)/Divorciado(a) & 52,3 & $0,8(0,6-1,1)$ & & $0,9(0,7-1,2)$ & \\
\hline Solteiro(a) & 73,7 & $1,2(0,9-1,4)$ & & $1,1(0,9-1,4)$ & \\
\hline Renda familiar per capita (salários mínimos) & & & 0,01 * & & 0,07 * \\
\hline Sem renda & 53,3 & 1,0 & & 1,0 & \\
\hline Até 1 & 68,0 & $1,3(0,9-1,8)$ & & $1,2(0,9-1,7)$ & \\
\hline $1,01-2$ & 73,6 & $1,4(0,9-2,0)$ & & $1,3(0,9-1,8)$ & \\
\hline $2,01-3$ & 66,7 & $1,3(0,7-2,2)$ & & $1,0(0,5-2,0)$ & \\
\hline 3 ou + & 90,0 & $1,7(1,1-2,5)$ & & $1,6(1,1-2,2)$ & \\
\hline \multicolumn{6}{|l|}{ Nível 2} \\
\hline Trabalho remunerado no último mês & & & 0,25 & & 0,51 \\
\hline Não & 68,5 & 1,0 & & 1,0 & \\
\hline Sim & 54,5 & $0,8(0,54-1,17)$ & & $0,9(0,61-1,28)$ & \\
\hline
\end{tabular}

(continua)

servaram maiores percentuais de sedentarismo entre os homens.

A orientação para atividade física por parte de profissionais de saúde das UBS foi realizada apenas por cerca de um quarto desses profissionais, o que provavelmente é insuficiente para promover alterações na atitude das pessoas em relação à prática de atividade física. Isso evidencia que as UBS no Estado de Pernambuco não estão atendendo as necessidades dos usuários em relação à orientação à prática da atividade física como instrumento de promoção da saúde. 
Tabela 3 (continuação)

\begin{tabular}{|c|c|c|c|c|c|}
\hline \multirow[t]{3}{*}{ Variáveis } & \multirow[t]{3}{*}{$\%$} & \multicolumn{2}{|c|}{ Análise bruta } & \multicolumn{2}{|c|}{ Análise ajustada } \\
\hline & & RP & Valor & RP & Valor \\
\hline & & (IC95\%) & de $p$ & (IC95\%) & de $p$ \\
\hline \multicolumn{6}{|l|}{ Nível 3} \\
\hline Autopercepção da saúde & & & 0,01 * & & 0,02 * \\
\hline Excelente & 61,5 & 1,0 & & 1,0 & \\
\hline Muito boa & 33,3 & $0,9(0,6-1,3)$ & & $0,8(0,6-1,3)$ & \\
\hline Boa & 61,4 & $0,5(0,2-1,0)$ & & $0,4(0,2-1,1)$ & \\
\hline Regular & 71,5 & $0,9(0,7-1,0)$ & & $0,9(0,8-1,0)$ & \\
\hline Ruim & 71,2 & $1,0(0,9-1,1)$ & & $1,0(0,9-1,2)$ & \\
\hline Identificação de atividade física & & & 0,12 & & 0,40 \\
\hline \multicolumn{6}{|l|}{ como benefício para a saúde } \\
\hline Não & 68,7 & 1,0 & & 1,0 & \\
\hline Sim & 62,4 & $0,9(0,8-1,0)$ & & $0,9(0,8-1,1)$ & \\
\hline \multicolumn{6}{|l|}{ Nível 4} \\
\hline Prescrição de atividade física no último ano & & & 0,05 & & 0,26 \\
\hline Não & 70,9 & 1,0 & & 1,0 & \\
\hline Sim & 62,4 & $0,9(0,8-1,0)$ & & $0,9(0,8-1,1)$ & \\
\hline
\end{tabular}

RP: razão de prevalência; IC95\%: intervalo de 95\% de confiança.

Nota: níveis correspondentes ao nível da variável no modelo hierárquico. Cada associação foi ajustada para as variáveis de mesmo nível.

* Teste de tendência;

** Segundo classificação da Associação Brasileira de Empresas de Pesquisa (http://www.abep.org/codigosguias/abep.cceb. pdf, acessado em 12/Jun/2007).

Essas observações sugerem que são necessários projetos e ações estruturadas e participativas entre os profissionais e população, além de investimentos por parte da atenção básica com objetivo de qualificar e reorganizar o sistema.

Até onde vai o conhecimento dos autores, esse é o primeiro estudo no Estado de Pernambuco que procura investigar o nível de sedentarismo entre populações de adultos e idosos residentes em áreas de abrangência de diferentes tipos de atendimentos à saúde: unidade básica tradicional e PSF. O percentual de sedentarismo foi semelhante entre os adultos e idosos assistidos com ou sem modelo PSF. Isso pode significar que o enfoque de uma maior valorização para a saúde pelo PSF, nas localidades estudadas, não esteja sendo atingido, seja por uma menor captação de indivíduos ou uma maior relutância, por motivos culturais ou outros, na aceitação das orientações direcionadas à saúde pela equipe da unidade de saúde. Ressalte-se ainda que o instrumento de avaliação de atividade física empregado, o IPAQ, é recomendado para indivíduos de 18-64 anos de idade muito embora venha sendo aplicado com freqüência em populações de idosos 14,24. Por outro lado, a associação positiva de autopercepção de saúde com maior nível de atividade física pode indicar que as pessoas mais ativas classifi- cam sua saúde de forma mais positiva do que os sedentários 26.

Podemos apontar ainda como vantagens do presente estudo uma casuística que incluiu todas as regiões do Estado de Pernambuco, zona litorânea, mata, agreste e sertão, com cidades de médio ou grande porte (com mais de 100 mil habitantes). Outro aspecto importante foi a utilização do IPAQ, que avalia os quatro componentes da atividade física (lazer, ocupação, transporte e trabalho domiciliar). Isso possibilitou a comparabilidade de nossos dados com outros estudos. As pesquisas que aferem apenas a atividade física de lazer tendem a subestimar o nível de atividade física das populações de baixa renda, como a aqui estudada. Ressalte-se ainda que o poder estatístico foi suficiente para análises brutas e ajustadas, permitindo que diferenças relevantes fossem identificadas.

O estudo apresenta algumas limitações. Inicialmente, nossos dados não devem ser extrapolados para a população geral, pois estudamos além de uma população de baixa renda, adstrita à UBS, que está principalmente instalada em locais de menor nível sócio-econômico. Além disso, a versão curta do IPAQ apesar de oferecer exemplos de perguntas de atividade física como meios de transporte, lazer e esportes, serviços domésticos 
Prevalência de sedentarismo, análise bruta e ajustada em relação ao desfecho sedentarismo em adultos, na população da área de abrangência de unidades básicas de saúde (UBS) do Estado de Pernambuco, Brasil. UBS tradicional.

\begin{tabular}{|c|c|c|c|c|c|}
\hline \multirow[t]{2}{*}{ Variáveis } & \multirow[t]{2}{*}{$\%$} & \multicolumn{2}{|c|}{ Análise bruta } & \multicolumn{2}{|c|}{ Análise ajustada } \\
\hline & & $\begin{array}{c}\text { RP } \\
\text { (IC95\%) }\end{array}$ & $\begin{array}{l}\text { Valor } \\
\text { de } p\end{array}$ & $\begin{array}{c}\text { RP } \\
\text { (IC95\%) }\end{array}$ & $\begin{array}{l}\text { Valor } \\
\text { de } p\end{array}$ \\
\hline \multicolumn{6}{|l|}{ Nível 1} \\
\hline Sexo & & & 0,10 & & 0,03 \\
\hline Masculino & 45,0 & 1,0 & & 1,0 & \\
\hline Feminino & 35,6 & $0,8(0,6-1,0)$ & & $0,7(0,5-1,0)$ & \\
\hline Idade (anos) & & & 0,01 * & & 0,10 * \\
\hline $30-40$ & 36,2 & 1,0 & & 1,0 & \\
\hline $41-50$ & 30,2 & $0,8(0,6-1,2)$ & & $0,8(0,5-1,2)$ & \\
\hline $51-64$ & 54,0 & $1,5(1,1-2,1)$ & & $1,3(0,9-1,8)$ & \\
\hline Cor da pele & & & 0,01 & & 0,02 \\
\hline Branca & 48,0 & 1,0 & & 1,0 & \\
\hline Não branca & 32,0 & $0,7(0,5-0,9)$ & & $0,7(0,5-1,0)$ & \\
\hline Escolaridade & & & 0,08 * & & 0,005 * \\
\hline Não estudou & 42,3 & 1,0 & & 1,0 & \\
\hline Fundamental incompleto & 46,0 & $1,1(0,7-1,6)$ & & $1,0(0,7-1,4)$ & \\
\hline Fundamental completo & 38,5 & $0,9(0,5-1,6)$ & & $0,6(0,3-1,2)$ & \\
\hline Médio incompleto & 38,9 & $0,9(0,5-1,8)$ & & $0,7(0,4-1,4)$ & \\
\hline Médio completo & 25,4 & $0,6(0,3-1,0)$ & & $0,4(0,2-0,8)$ & \\
\hline Superior & 50,0 & $1,2(0,6-2,2)$ & & $0,8(0,4-1,8)$ & \\
\hline Tabagismo & & & 0,95 * & & 0,24 * \\
\hline Não, nunca fumou & 41,9 & 1,0 & & 1,0 & \\
\hline Já fumou & 32,4 & $0,8(0,5-1,1)$ & & $0,6(0,4-1,0)$ & \\
\hline Fumante atual & 43,9 & $1,0(0,8-1,5)$ & & $0,9(0,6-1,2)$ & \\
\hline Padrão de consumo sócio-econômico ** & & & 0,28 * & & 0,02 * \\
\hline A, B ou C & 43,6 & 1,0 & & 1,0 & \\
\hline $\mathrm{D}$ & 38,8 & $0,9(0,6-1,2)$ & & $0,7(0,5-1,1)$ & \\
\hline$E$ & 36,0 & $0,8(0,6-1,2)$ & & $0,6(0,4-0,9)$ & \\
\hline Situação conjugal & & & 0,72 & & 0,60 \\
\hline Casado(a)/Com companheiro(a) & 41,6 & 1,0 & & 1,0 & \\
\hline Viúvo(a) & 34,6 & $0,8(0,5-1,4)$ & & $0,8(0,4-1,4)$ & \\
\hline Separado(a)/Divorciado(a) & 31,7 & $0,8(0,5-1,2)$ & & $0,8(0,5-1,3)$ & \\
\hline Solteiro(a) & 44,4 & $1,1(0,7-1,6)$ & & $1,3(0,9-2,0)$ & \\
\hline Renda familiar per capita (salários mínimos) & & & 0,35 * & & 0,74 * \\
\hline Sem renda & 56,2 & 1,0 & & 1,0 & \\
\hline Até 1 & 36,0 & $0,6(0,4-0,9)$ & & $0,7(0,5-1,0)$ & \\
\hline $1,01-2$ & 38,8 & $0,7(0,4-1,1)$ & & $0,7(0,4-1,2)$ & \\
\hline $2,01-3$ & 44,4 & $0,8(0,4-1,7)$ & & $0,6(0,3-1,6)$ & \\
\hline 3 ou + & 100,0 & $1,8(1,3-2,4)$ & & $1,4(0,8-2,5)$ & \\
\hline \multicolumn{6}{|l|}{ Nível 2} \\
\hline Trabalho remunerado no último mês & & & 0,45 & & 0,34 \\
\hline Não & 41,9 & 1,0 & & 1,0 & \\
\hline Sim & 37,6 & $0,9(0,7-1,2)$ & & $0,9(0,6-1,2)$ & \\
\hline
\end{tabular}

(continua) 
Tabela 4

\begin{tabular}{|c|c|c|c|c|c|}
\hline \multirow[t]{2}{*}{ Variáveis } & \multirow[t]{2}{*}{$\%$} & \multicolumn{2}{|c|}{ Análise bruta } & \multicolumn{2}{|c|}{ Análise ajustada } \\
\hline & & $\begin{array}{c}\text { RP } \\
\text { (IC95\%) }\end{array}$ & $\begin{array}{l}\text { Valor } \\
\text { de } p\end{array}$ & $\begin{array}{c}\text { RP } \\
\text { (IC95\%) }\end{array}$ & $\begin{array}{l}\text { Valor } \\
\text { de p }\end{array}$ \\
\hline \multicolumn{6}{|l|}{ Nível 3} \\
\hline Autopercepção da saúde & & & 0,96 * & & 0,84 * \\
\hline Excelente & 47,6 & 1,0 & & 1,0 & \\
\hline Muito boa & 36,4 & $0,8(0,3-1,9)$ & & $0,7(0,3-2,1)$ & \\
\hline Boa & 38,9 & $0,8(0,5-1,4)$ & & $0,9(0,5-1,5)$ & \\
\hline Regular & 38,5 & $0,8(0,5-1,3)$ & & $0,8(0,5-1,5)$ & \\
\hline Ruim & 46,9 & $1,0(0,6-1,8)$ & & $1,1(0,6-2,1)$ & \\
\hline Identificação de atividade física & & & 0,34 & & 0,51 \\
\hline \multicolumn{6}{|l|}{ como benefício para a saúde } \\
\hline Não & 43,6 & 1,0 & & 1,0 & \\
\hline Sim & 38,1 & $0,9(0,7-1,1)$ & & $0,9(0,7-1,2)$ & \\
\hline \multicolumn{6}{|l|}{ Nível 4} \\
\hline Prescrição de atividade física no último ano & & & 0,39 & & 0,80 \\
\hline Não & 39,3 & 1,0 & & 1,0 & \\
\hline Sim & 31,6 & $0,8(0,5-1,3)$ & & $0,8(0,5-1,3)$ & \\
\hline
\end{tabular}

RP: razão de prevalência; IC95\%: intervalo de 95\% de confiança.

Nota: níveis correspondentes ao nível da variável no modelo hierárquico. Cada associação foi ajustada para as variáveis de mesmo nível.

* Teste de tendência;

** Segundo classificação da Associação Brasileira de Empresas de Pesquisa (http://www.abep.org/codigosguias/abep.cceb. pdf, acessado em 12/Jun/2007).

Tabela 5

Prevalência de sedentarismo, análise bruta e ajustada em relação ao desfecho sedentarismo em adultos, na população da área de abrangência de unidades básicas de saúde (UBS) do Estado de Pernambuco, Brasil. UBS com Programa Saúde da Família (PSF).

\begin{tabular}{|c|c|c|c|c|c|}
\hline \multirow[t]{2}{*}{ Variáveis } & \multirow[t]{2}{*}{$\%$} & \multicolumn{2}{|c|}{ Análise bruta } & \multicolumn{2}{|c|}{ Análise ajustada } \\
\hline & & $\begin{array}{c}\text { RP } \\
\text { (IC95\%) }\end{array}$ & $\begin{array}{l}\text { Valor } \\
\text { de } p\end{array}$ & $\begin{array}{c}\text { RP } \\
\text { (IC95\%) }\end{array}$ & $\begin{array}{l}\text { Valor } \\
\text { de } p\end{array}$ \\
\hline \multicolumn{6}{|l|}{ Nível 1} \\
\hline Sexo & & & $<0,001$ & & $<0,001$ \\
\hline Masculino & 44,5 & 1,0 & & 1,0 & \\
\hline Feminino & 27,7 & $0,6(0,5-08)$ & & $0,6(0,5-0,8)$ & \\
\hline Idade (anos) & & & 0,16 * & & 0,15 * \\
\hline $30-40$ & 33,6 & 1,0 & & 1,0 & \\
\hline $41-50$ & 33,7 & $1,0(0,8-1,3)$ & & $1,0(0,8-1,3)$ & \\
\hline $51-64$ & 40,3 & $1,2(0,9-1,5)$ & & $1,2(0,9-1,5)$ & \\
\hline Cor da pele & & & 0,86 & & 0,74 \\
\hline Branca & 36,0 & 1,0 & & 1,0 & \\
\hline Não branca & 35,3 & $1,0(0,8-1,2)$ & & $1,0(0,8-1,2)$ & \\
\hline Escolaridade & & & 0,83 * & & 0,86 * \\
\hline Não estudou & 34,8 & 1,0 & & 1,0 & \\
\hline Fundamental incompleto & 35,8 & $1,0(0,8-1,4)$ & & $1,0(0,76-1,36)$ & \\
\hline Fundamental completo & 40,9 & $1,2(0,8-1,7)$ & & $1,2(0,79-1,73)$ & \\
\hline Médio incompleto & 29,6 & $0,8(0,5-1,6)$ & & $0,9(0,46-1,66)$ & \\
\hline Médio completo & 33,9 & $1,0(0,7-1,4)$ & & $0,9(0,6-1,4)$ & \\
\hline Superior & 44,4 & $1,3(0,8-2,1)$ & & $1,0(0,8-1,4)$ & \\
\hline
\end{tabular}

(continua) 
Tabela 5 (continuação)

\begin{tabular}{|c|c|c|c|c|c|}
\hline \multirow[t]{2}{*}{ Variáveis } & \multirow[t]{2}{*}{$\%$} & \multicolumn{2}{|c|}{ Análise bruta } & \multicolumn{2}{|c|}{ Análise ajustada } \\
\hline & & $\begin{array}{c}\mathrm{RP} \\
(1 \mathrm{O} 5 \%)\end{array}$ & Valor & $\begin{array}{c}\mathrm{RP} \\
\end{array}$ & Valor \\
\hline \multicolumn{6}{|l|}{ Nível 1} \\
\hline Tabagismo & & & 0,78 * & & 0,89 * \\
\hline Não, nunca fumou & 34,9 & 1,0 & & 1,0 & \\
\hline Já fumou & 37,3 & $1,1(0,8-1,4)$ & & $1,0(0,86-1,2)$ & \\
\hline Fumante atual & 35,8 & $1,0(0,8-1,3)$ & & $1,0(0,7-1,3)$ & \\
\hline Padrão de consumo sócio-econômico ** & & & 0,30 * & & 0,44 * \\
\hline A, B ou C & 40,1 & 1,0 & & 1,0 & \\
\hline $\mathrm{D}$ & 33,0 & $0,8(0,6-1,1)$ & & $0,9(0,7-1,1)$ & \\
\hline $\mathrm{E}$ & 35,7 & $0,9(0,7-1,1)$ & & $0,9(0,7-1,2)$ & \\
\hline Situação conjugal & & & 0,92 & & 0,90 \\
\hline Casado(a)/Com companheiro(a) & 36,0 & 1,0 & & 1,0 & \\
\hline Viúvo(a) & 34,9 & $1,0(0,6-1,5)$ & & $1,1(0,7-1,8)$ & \\
\hline Separado(a)/Divorciado(a) & 33,9 & $0,9(0,6-1,4)$ & & $1,0(0,7-1,4)$ & \\
\hline Solteiro(a) & 36,5 & $1,0(0,7-1,4)$ & & $1,0(0,7-1,5)$ & \\
\hline Renda familiar per capita (salários mínimos) & & & 0,60 * & & 0,91 * \\
\hline Sem renda & 42,9 & 1,0 & & 1,0 & \\
\hline Até 1 & 34,2 & $0,8(0,6-1,1)$ & & $0,7(0,5-1,0)$ & \\
\hline $1,01-2$ & 36,5 & $0,8(0,6-1,3)$ & & $0,7(0,5-1,1)$ & \\
\hline $2,01-3$ & 33,3 & $0,8(0,3-1,8)$ & & $0,7(0,3-1,6)$ & \\
\hline 3 ou + & 57,1 & $1,3(0,8-2,3)$ & & $1,1(0,6-2,1)$ & \\
\hline \multicolumn{6}{|l|}{ Nível 2} \\
\hline Trabalho remunerado no último mês & & & 0,61 & & \\
\hline Não & & 1,0 & & 1,0 & 0,48 \\
\hline Sim & & $1,1(0,9-1,3)$ & & $0,9(0,74-1,15)$ & \\
\hline \multicolumn{6}{|l|}{ Nível 3} \\
\hline Autopercepção da saúde & & & 0,04 * & & 0,01 * \\
\hline Excelente & 29,0 & 1,0 & & 1,0 & \\
\hline Muito boa & 26,5 & $0,9(0,4-2,0)$ & & $1,0(0,5-2,3)$ & \\
\hline Boa & 33,2 & $1,1(0,6-2,0)$ & & $1,2(0,7-2,3)$ & \\
\hline Regular & 37,4 & $1,3(0,7-2,3)$ & & $1,5(0,8-2,7)$ & \\
\hline Ruim & 42,5 & $1,5(0,8-2,7)$ & & $1,7(0,9-3,2)$ & \\
\hline Identificação de atividade física & & & 0,62 & & 0,51 \\
\hline \multicolumn{6}{|l|}{ como benefício para a saúde } \\
\hline Não & 36,9 & 1,0 & & 1,0 & \\
\hline Sim & 35,0 & $0,9(0,8-1,2)$ & & $0,9(0,8-1,1)$ & \\
\hline \multicolumn{6}{|l|}{ Nível 4} \\
\hline Prescrição de atividade física no último ano & & & 0,88 & & 0,70 \\
\hline Não & 34,2 & 1,0 & & 1,0 & \\
\hline Sim & 34,9 & $1,0(0,8-1,3)$ & & $1,0(0,8-1,4)$ & \\
\hline
\end{tabular}

RP: razão de prevalência; IC95\%: intervalo de 95\% de confiança.

Nota: níveis correspondentes ao nível da variável no modelo hierárquico. Cada associação foi ajustada para as variáveis de mesmo nível.

* Teste de tendência;

** Segundo classificação da Associação Brasileira de Empresas de Pesquisa (http://www.abep.org/codigosguias/abep.cceb. pdf, acessado em 12/Jun/2007). 
e outros, não oferece uma avaliação mais precisa do nível de atividade física nesses quatro domínios como a versão longa do IPAQ. Deve ser registrado ainda que a taxa de não-resposta, $7,1 \%$ entre os adultos e $7,5 \%$ nos idosos, incluiu não só as pessoas selecionadas que se recusaram a responder o questionário, como também os domicílios nos quais não foram encontrados os residentes, o que pode gerar uma superestimativa do percentual de não-respondentes por livre recusa. Outra limitação do estudo foi a ausência de diferenciação entre pardos e pretos.

Em conclusão, os níveis de sedentarismo em populações de áreas de abrangência das UBS no Estado de Pernambuco são elevados, quando comparados com a Região Sul do Brasil. O combate ao sedentarismo, como um importante fator de risco para as doenças crônicas não transmissíveis, deve ser prioritário, especialmente para as populações de baixa renda, em que essas afecções vêm rapidamente aumentando de prevalência. Dessa forma, se fazem necessárias políticas específicas na estratégia da atenção primária no sentido de priorizar a atividade física na assistência à saúde das populações adultas e idosas. Acreditamos ainda que a presença de novos profissionais da área da saúde, como educadores físicos e nutricionistas, no grupo profissional que atende a atenção básica colaboraria muito no aumento dos níveis de orientação e possivelmente na modificação do comportamento sedentário da população.

\section{Resumo}

A transição epidemiológica no Brasil, em parte, é explicada pelos baixos níveis de atividade física da população. Entretanto, poucos estudos têm explorado atividade física em populações de baixa renda. Nesta realidade, as unidades básicas de saúde (UBS) ganham importância. Em estudo com delineamento transversal, avaliou-se a prevalência de sedentarismo e fatores associados em 1.018 adultos e 1.010 idosos, residentes em áreas de UBS de dez municípios com mais de 100 mil habitantes no Estado de Pernambuco, Brasil. A prevalência de sedentarismo foi de 37,18\% (IC95\%: 34,0-40,2) para adultos e de 68,3\% (IC95\%: 65,3-71,3) para idosos. A identificação da prática de atividade física como um dos fatores mais importantes para a manutenção da saúde esteve associado com sedentarismo no grupo dos idosos. Foi verificado um menor percentual de sedentarismo entre adultos associado com uma autopercepção de saúde satisfatória. Conclui-se que a prevalência de sedentarismo em áreas de abrangência das UBS em Pernambuco é mais elevada quando comparada com a Região Sul.

Atividade Motora; Centros de Saúde; Adulto; Idoso

\section{Colaboradores}

J. G. B. Alves participou da concepção do artigo e de todas as fases até a redação final. F. V. Siqueira colaborou na concepção do artigo, análises de dados, interpretação e redação final; coordenou o trabalho de campo do estudo e participou da elaboração de instrumentos e processamento dos dados. J. N. Figueiroa foi responsável pelas análises e interpretações dos resultados. L. A. Facchini contribuiu na revisão do artigo, foi o coordenador geral do projeto em todas as suas fases. D. S. Silveira participou da concepção do artigo, e como revisora em todas as fases da elaboração do artigo. R. X. Piccini colaborou na revisão final do artigo e nas relações do tema com a atenção básica. E. Tomasi contribuiu na revisão do artigo. E. Thumé colaborou na elaboração e revisão do artigo e na elaboração de tabelas. P. C. Hallal participou da revisão do trabalho final na parte relacionada à atividade física.

\section{Agradecimentos}

Ao Ministério da Saúde e Banco Mundial pelo financiamento. 


\section{Referências}

1. Warburton DE, Katzmarzyk PT, Rhodes RE, Shephard RJ. Evidence-informed physical activity guidelines for Canadian adults. Can J Public Health 2007; 98 Suppl 2:S16-68.

2. Allender S, Foster C, Scarborough P, Rayner M. The burden of physical activity-related ill health in the UK. J Epidemiol Community Health 2007; 61:344-8.

3. World Health Organization. Physical inactivity: a global public health problem. http://www.who. int/dietphysicalactivity/factsheet_inactivity/en/ (acessado em 12/Abr/2008).

4. Garrett NA, Brasure M, Schmitz KH, Schultz MM, Huber MR. Physical inactivity: direct cost to a health plan. Am J Prev Med 2004; 27:304-9.

5. Organização Mundial da Saúde. Estratégia global em alimentação saudável, atividade física e saúde. http://dtr2004.saude.gov.br/nutricao/documen tos/eb_portugues.pdf (acessado em 08/Abr/2008).

6. Popkin BM, Gordon-Larsen P. The nutrition transition: worldwide obesity dynamics and their determinants. Int J Obes Relat Metab Disord 2004; 28 Suppl 3:S2-9.

7. Monteiro CA, Moura EC, Conde WL, Popikn BM. Socioeconomic status and obesity in adult populations of developing countries: a review. Bull World Health Organ 2004; 82:940-6.

8. Filozof C, Gonzalez C, Sereday M, Mazza C, Braguinsky J. Obesity prevalence and trends in LatinAmerican countries. Obes Rev 2001; 2:99-106.

9. Hallal PC, Dumith SC, Bastos JP, Reichert FF, Siqueira FV, Azevedo MR. Evolução da pesquisa epidemiológica em atividade física no Brasil: revisão sistemática. Rev Saúde Pública 2007; 41:453-60.

10. Fuscaldo JM. Prescribing physical activity in primary care. WV Med J 2002; 98:250-3.

11. Siqueira FV, Facchini LA, Piccini RX, Tomasi E, Thumé L, Silveira DS, et al. Atividade física em adultos e idosos residentes em áreas de abrangência de unidades básicas de saúde de municípios das regiões Sul e Nordeste do Brasil. Cad Saúde Pública 2008; 24:39-54.

12. Ministério da Saúde. Termo de referência para o estudo de linha de base nos municípios selecionados para o componente 1 do PROESF. Brasília: Ministério da Saúde; 2004.

13. Craig CL, Marshall AL, Sjostrom M, Bauman AE, Booth ML, Ainsworth BE, et al. International physical activity questionnaire: 12-country reliability and validity. Med Sci Sports Exerc 2003; 35: 1381-95.

14. Barros MV, Nahas MV. Comportamentos de risco, auto-avaliação do nível de saúde e percepção de estresse entre trabalhadores da indústria. Rev Saúde Pública 2001; 35:554-63.

15. Barros AJ, Hirakata VN. Alternatives for logistic regression in cross-sectional studies: an empirical comparison of models that directly estimate the prevalence ratio. BMC Med Res Methodol 2003; $3: 21$.
16. United States Department of Health and Human Services (USDHHS). Physical activity patterns in modern culture. http://www.enotes.com/publichealth-encyclopedia/physical-activity (acessado em 26/Set/2009).

17. Victora CG, Huttly SR, Fuches SC, Olinto MT. The role of conceptual frameworks in epidemiological analysis: a hierarquical approach. Int J Epidemiol 1997; 26:224-7.

18. Facchini LA, Piccini RX, Tomasi E, Thumé E, Silveira DS, Siqueira FV, et al. Desempenho do PSF no Sul e no Nordeste do Brasil: avaliação institucional e epidemiológica da atenção básica à saúde. Ciênc Saúde Coletiva 2006; 11:669-81.

19. Facchini LA, Piccini RX, Tomasi E, Thumé E, Teixeira VA, Silveira DS, et al. Avaliação de efetividade da atenção básica à saúde em municípios das regiões Sul e Nordeste do Brasil: contribuições metodológicas. Cad Saúde Pública 2008; 24 Suppl 1:S159-72.

20. Secretaria de Gestão Estratégica e Participativa, Secretaria de Vigilância em Saúde, Ministério da Saúde. VIGITEL Brasil 2008: vigilância de fatores de risco e proteção para doenças crônicas por inquérito telefônico. Brasília: Ministério da Saúde; 2009. (Série G. Estatística e Informação em Saúde).

21. Hallal PC, Victora CG, Wells JC, Lima RC. Physical inactivity: prevalence and associated variables in Brazilian adults. Med Sci Sports Exerc 2003; 35:1894-900.

22. Matsudo SM, Matsudo VR, Araújo T, Andrade D, Andrade E, Oliveira L, et al. Nível de atividade física da população do Estado de São Paulo: análise de acordo com o gênero, idade, nível socioeconômico, distribuição geográfica e de conhecimento. Rev Bras Ciênc Mov 2002; 10:41-50.

23. Alves JG, Gale CR, Mutrie N, Correia JB, Batty GD. A 6-month exercise intervention among inactive and overweight favela-residing women in Brazil: the Caranguejo Exercise Trial. Am J Public Health 2009; 99:76-80.

24. Van Der Horst K, Paw MJ, Twisk JW, Van Mechelen W. A brief review on correlates of physical activity and sedentariness in youth. Med Sci Sports Exerc 2007; 39:1241-50.

25. Hollmann W, Strüder HK, Tagarakis CV, King G. Physical activity and the elderly. Eur J Cardiovasc Prev Rehabil 2007; 14:730-9.

26. Benedetti TRB, Borges LJ, Petroski EL, Gonçalves LHT. Atividade física e estado de saúde mental de idosos. Rev Saúde Pública 2008; 42:302-7.

27. Baretta E, Baretta M, Peres KG. Nível de atividade física e fatores associados em adultos no Município de Joaçaba, Santa Catarina, Brasil. Cad Saúde Pública 2007; 23:1595-602.

Recebido em 12/Mar/2009

Versão final reapresentada em 05/Nov/2009

Aprovado em 10/Nov/2009 